\title{
ÉVALUATION DU PROCESSUS DANS UNE EXPÉRIENCE DE GROUPE POUR PARENTS NÉGLIGENTS
}

\author{
ERCILIA PALACIO-QUINTIN et SYLVIE CALILLE \\ Université du Québec à Trois-Rivières
}

\begin{abstract}
RÉSUMÉ
Dans cet articie, nous rapportons une évaluation du processus d'une expérience de groupe avec des parents négligents. Les parents négligents éprouvent évidemment des difficultés à assumer leurs responsabilités parentales, mais ils présentent aussi comme caractéristique de vivre des situations difficiles, de souffrir d'isolement, et d'étre peu intégrés dans leur communautés. Une expérience de groupe a été réalisée auprès de 35 familles, dont 33 femmes et 17 hommes, retenues comme négligentes par le CPEJ. Les parents ont été répartis en quatre groupes chacun animé par un professionnel. Nous tentons ici de dégager ce que l'expérience de groupe comporte et peut apporter compte tenu des besoins spécifiques de ces parents.
\end{abstract}

Les 20 dernières années ont vu grandir l'intérêt à l'égard des enfants maltraités et de nombreuses recherches ont tenté de décrire les caractéristiques de l'enfant maltraité et du parent maltraitant. Mais tous les aspects de la maltraitance n'ont pas fait l'objet du même intérêt. Ainsi, la négligence a reçu moins d'attention et ce malgré son haut taux d'incidence (Palacio-Quintin \& Éthier, 1993). Les recherches touchant les modalités d'intervention auprès des familles négligentes sont encore plus rares. À la suite d'une revue de la littérature, Gaudin (1993) constate qu'il y a un nombre très réduit d'études sur l'intervention auprès des familles maltraitantes qui différencient les familles négligentes par rapport à des familles présentant d'autres types de maltraitance.

Sur le plan de la pratique, des progrès importants ont été accomplis quant à l'identification des enfants maltraités et à leur prise en charge par le système de protection. Mais il subsiste encore d'importantes défaillances surtout dans les réponses apportées aux besoins des familles négligentes. Les récidives sont très nombreuses et les intervenants se sentent souvent désemparés face à cette clientèle.

La conceptualisation et l'expérimentation de nouvelles formes d'intervention auprès des familles négligentes ainsi que des recherches pour vérifier leur efficacité et évaluer leur processus d'implantation constituent donc une urgence autant sociale que scientifique. Compte tenu de cet état de fait, à partir d'une étude exhaustive des caractéristiques particulières des familles négligentes, nous avons

\footnotetext{
L'ensemble de la recherche portant sur l'intervention auprès des familles négligentes a été rendu possit le sràce à une subvention de Bien-ètre et santé Canada (Division de la prévention de la violence familiale). Les demandes de tirés a part et d'information supplëmentaire peuvent être adressées à: E. PalacioQuintin, GREDEF, Univensité du Quebec à Trois-Rivières, C.P. 500, Trois-Rivières, PQ G9A 5 H7.
} 
développé (Palacio-Quintin, Éthier. Jourdan-lonescu, \& Lacharité, 1995) le Pro. gramme d'aide personnelle, familiale et communautaire (PAPFC) qui tente de répondre aux multiples problèmes des familles négligentes. Une recherche a ćté menée afin d'expérimenter le PAPFC et d'en évaluer les effects chez la clientète visée. L'expérimentation a été réalisée en étroite collaboration avec les services du Centre de protection de l'enfance et de la jeunesse Mauricie-Bois-Francs (CPE]. MBF). Le programme a été expérimenté auprès des familles présentant des comportements de négligence majeurs envers leurs jeunes enfants. La description complète du programme, les aspects méthodologiques de la recherche, les procédures utilisées, l'analyse du processus, et les données sur l'impact de l'intervention sur la clientèle ont été présentés dans le rapport final de la recherche (PalacioQuintin, Couture, \& Paquet, 1995). Le PAPFC est un programme multidimensionnel qui tente d'agir autant sur certaines dimensions personnelles du parent négligent que sur les dimensions familiales et sociales. Diverses techniques sont utilisées dans le processus d'intervention pour atteindre les objectifs du programme. Une des techniques intégrée dans l'ensemble est l'activité de groupe des parents. Les techniques de groupe ont en effet une longue tradition dans divers secteurs de la psychologie et de l'animation. Pourtant, elles ont été jusqu'à ce jour peu utilisées et explorées avec des familles négligentes.

Le présent article a comme but de présenter une analyse de l'expérience de groupe réalisée à l'intérieur de la recherche précitée. Avant d'élaborer davantage sur cette question, nous procéderons d'abord à une définition de la négligence et à une brève description des caractéristiques et des besoins particuliers des familles négligentes et violentes, besoins et caractéristiques qui doivent être considérés en vue de l'intervention. Un aperçu de certains aspets de l'intervention auprès des familles négligentes complétera les éléments qui nous amènent à poser des questions spécifiques au sujet de l'activité de groupe avec ces parents.

\section{LA NÉGLIGENCE ET LES CARACTÉRISTIQUES DES FAMILLES NÉGLIGENTES}

La négligence est une forme de mauvais traitement caractérisée par un manque de soins aux plans de la santé, de l'hygiène corporelle, de l'alimentation, de la surveillance, de l'éducation, ou des besoins affectifs, mettant en péril le développement normal de l'enfant (Polansky, Chalmers, Williams, \& Buttenwieser. 1981). Il s'agit d'une carence des soins nécessaires pour répondre aux besoins de base de l'enfant selon son âge et son niveau de développement. La négligence ne peut done être définie que si l'on tient compte des besoins spécifiques de l'enfant (Palacio-Quintin \& Éthier, 1993). Cette forme de maltraitance est caractérisée par l'omission ou un manque de gestes de la part du parent envers l'enfant. L'abus physique et émotif se caractérise par contre par des actes d'agression physique ou émotif envers l'enfant. Le parent violent agit de façon inadéquate alors que le parent négligent omet d'agir, n'entreprend pas des actions qui sont inhérentes au rôle parental. Le parent violent se «bats, tandis que le parent négligent démissionne.

Les risques de négligence sont plus grands lorsque l'enfant est jeune et plus dépendant. On constate que les parents des très jeunes enfants présentent 
davantage des comportements de négligence que d'abus physique. Mais dans bon nombre de cas, la négligence s'accompagne de violence. Dans ces cas la violence apparait de façon épisodique et s'inscrit sur un fond de négligence et de désorganisation familiale. Elle correspond à une dynamique familiale très différente de celle des familles violentes. Qu'il y ait ou non des épisodes de violence qui accompagnent la négligence, celle-ci n'en demeure pas moins un phénomène spécifique qui doit être considéré en soi. Mais la négligence est un phénomène multiforme: elle peut varier autant par son type (alimentaire, éducative, etc.) que par șon intensité (đe légère à grave) ou sa durée (circonstancielle ou chronique).

Les facteurs liés à la négligence et les caractéristiques des familles négligentes qui ont pu être décrits sont aussi très variés.

La franche majorité des cas de négligence parentale retenus par les services de protection concerne les personnes les plus défavorisées de notre société: très pauvres, sans travail, et avec un très bas niveau d'éducation (Chamberland, Bouchard, \& Beaudry, 1986; Wolock \& Horowitz, 1979). Les observations montrent đ'ailleurs que les familles négligentes sont davantage défavorisées que les familles retenues par les services de protection pour violence envers leurs enfants (Crittenden, 1988; Giovannoni, 1971; Palacio-Quintin \& Éthier, 1993).

Les parents négligents sont également très isolés socialement. Cet isolement a été attribué par de nombreux études à l'écologie sociale, e'est-à-dire aux conditions environnementales dans lesquelles vivent ces familles (Chamberland et al., 1986; Garbarino \& Crouter, 1978). D'autres auteurs ont mis en relief qu'il faut aussi tenir compte d'une difficulté des mères négligentes à s'insérer dans un réseau social qui demande de la réciprocité (Polansky, Ammoms, \& Gaudin, 1985). La méfiance envers les autres est une caractéristique assez fréquente. Cette difficulté à établir des liens de réciprocité et de confiance peut être reliée aux antécédents de vie difficiles de ces personnes pendant leur propre enfance. Plusieurs de ces mères ont vécu de la violence, de la négligence, des abus sexuels, des situations familiales perturbées, et des placements en foyer d'accueil. Les pères ne sont malheureusement jamais considérés dans les études mentionnées. La récente étude de Lacharité (1995) sur un petit groupe de pères et de conjoints de familles négligentes laisse croire que les hommes de ces familles ont également un passé familial très perturbé. Les mères négligentes présentent également un niveau extrêmement élevé de dépression (Downey \& Coyne, 1990; Kinard, 1982), de stress parental (Éthier, 1992) et de stress lié à des situations de vie chroniques (Egeland, Breitenbucher, \& Rosenberg, 1980). Les hommes des familles négligentes présentent aussi un niveau de dépression sévère et de tendances dépressives plus élevé que les hommes de la population générale (Palacio-Quintin et al., 1995) mais moins élevé que chez les mères négligentes. Cette différence entre les sexes correspond à celle qui est constatée dans la population générale où deux fois plus de femmes que d'hommes présentent des manifestations de dépression majeurs (American Psychiatric Association, 1994).

Finalement, deux aspects ont été particulièrement signalés comme déficitaires chez les parents négligents: leurs habiletés et leurs comportements parentaux d'une part, et les fonctions cognitives servant à la résolution de problèmes, d'autre part. Les mères maltraitantes stimulent moins leur enfant et interagissent moins avec lui, elles ont plus d'interactions négatives (Twentyman \& Bousha, 1984), ne 
retirent pas de plaisir de leur contact avec l'enfant (Barth, 1985), et ont des attentes irréalistes face à lui (Wolfe, Edwards, Manion, \& Koverola, 1988). Le manque d'efficacité dans les tâches parentales contribue à augmenter le stress et la dé. pression des mères maltraitantes. Azar (1984) et Hansen, Palotta, Thishelman. Conaway, et McMillan (1989) ont révélé que les mères maltraitantes montrent une moindre habilité cognitive pour résoudre des problèmes relatifs à la vie quotidienne et à l'éducation de l'enfant que les mères non maltraitantes. Les travaux de notre groupe de recherche (Éthier, Palacio-Quintin, \& Couture, 1993) ont mis également en relief le fait que, parmi les mères négligentes, il y a une proportion élevée de femmes avec un niveau intellectuel inférieur à la moyenne non seulement de la population générale mais aussi de leur sous-groupe socio-économique de référence (mères pauvres non négligentes). Plus récemment, nous avons constatế le même phénomène chez les hommes des familles négligentes (Palacio-Quintin et al. 1995).

Soulignons enfin l'importance que l'on devrait accorder au rôle de l'homme au sein de la famille négligente. Bien que l'on possède peu de données scientifiques à cet égard puisque les écrits parlent seulement de la mère négligente, nos observations nous permettent de croire que l'on ne peut pas comprendre le phénomène et encore moins y apporter des solutions si on ne considère pas l'ensemble de la dynamique familiale et donc la participation ou la non participation du père ou du conjoint aux responsabilités familiales. Les écrits mentionnent fréquemment la présence d'un nombre élevé de mères monoparentales parmi les familles maltraitantes (Kimball, Steward, Conger, \& Burgess, 1980). Or, une récente étude (Palacio-Quintin, 1995) a permis de mettre en relief le fait que la majorité de ces mères vivaient une situation de violence conjugale peu de temps avant le signalement au CPEJ.

Il est possible de dire, en résumé, que les familles négligentes sont les plus démunies des démunies. En effet, elle ne sont pas démunies seulement du point de vue économique mais également du point de vue social et culturel. À ceci s'ajoute une absence de bien-être autant familial que personnel pour chaque membre.

\section{L'INTERVENTION AUPRÈS DES FAMILLES NÉGLIGENTES}

Compte tenu de la multiplicité des problèmes constatés chez les familles négligentes, bien de voies différentes peuvent être empruntées dans l'intervention. Les approches théoriques ainsi que les techniques utilisables sont également variées. Le domaine à explorer est done très vaste: approches d'entraînement aux habiletés parentales, interventions centrées sur la famille, interventions communautaires en vue d'améliorer le réseau social, suivis individuels de cas avec des techniques de counseling, etc. Compte tenu de la question traitée dans cet article, il est pertinent de s'attarder à deux aspects de l'intervention; les approches de groupe et l'entrainement aux habiletés parentales.

Plusieurs auteurs (Cohn, 1979; Daro, 1988) considèrent que l'utilisation d'approches de groupe avec les familles maltraitantes ameliore l'impact des interventions. Mais malgré ces considérations, peu de travaux se sont arrêtés à analyser 1 'impact des interventions de groupe chez les parents maltraitants et encore moins 
chez les parents négligents. Aderman et Russell (1990) rapportent une amélioration dans les soins apportés à leurs enfants par des parents abusifs et négligents, après une intervention en groupe de neuf semaines. Mais l'évaluation est basée sur les jugements qualitatifs non systématiques de thérapeutes et ce auprès d'un petit groupe de parents. En outre, les parents négligents ne sont pas distingués des parents abusifs. Landsman, Nelson, Allen, et Tyler (1992) sont les seuls à rapporter l'analyse d'une intervention de groupe auprès de parents négligents chroniques. Le Oregon Self-Sufficiency Project impliquait des activités de groupe pendant 24 semaines avec 31 familles. Des activités de groupe séparées pour les parents, les enfants, et les adolescents étaient suivies de rencontres de groupe de familles. L'emphase du projet étant sur l'empowerment de la famille, les activités du groupe parental étaient peu structurées. Une série d'autres services tels que des visites à domicile, des services de garderie pour offrir un répit aux parents, une thérapie individuelle, complètent l'intervention de groupe. Les auteurs rapportent que les familles ont assisté en moyenne à la moitié seulement des 24 rencontres de groupe. Des mesures d'observation montrent que seulement $45 \%$ des familles manifestent des améliorations significatives et que ces améliorations sont significativement corrélées avec le nombre de présences à l'activité de groupe.

Du côté de la formation aux habiletés parentales, les expériences et les études sont plus nombreuses. Une trentaine d'études d'intervention visant l'amélioration des habiletés parentales chez des parents maltraitants ou à risque a pu être relevée. Presque la moitié de ces études se réduisent à l'étude d'un ou de deux cas et plusieurs autres ne présentent pas d'évaluation systématique. Parmi les autres, nous trouvons des expériences avec des parents maltraitants (abusifs seulement ou abusifs et négligents confondus) ou à risque. Comme le constatent Wolfe et al, (1988), ces parents peuvent tirer bénéfice d'entrainements structurés en vue d'améliorer leur efficacité parentale. Un examen attentif des résultats des travaux nous permet de voir que c'est surtout une amélioration de la perception que la mère a d'elle-méme ou de l'enfant qui est le gain le plus important (Lovell, 1986; Wolfe, Sandler, \& Kaufman, 1981). Bien que l'on ait pu constater certains apprentissages pendant le déroulement de l'intervention, tous les auteurs soulignent par ailleurs le peu de durabilité de ces acquisitions et surtout l'absence de transfert de ces acquisitions à la vie quotidienne.

Même si la formation aux habiletés parentales a une longue tradition auprès des parents de la population générale, elle a longtemps laissé le père de côté (Bernal \& North, 1978; Croake \& Glover, 1977; Levant, 1988; Tavormina, 1974). Pourtant les résultats de l'étude de Price-Bonham et Skeen (1979) démontrent que les pères perçoivent leurs fonctions paternelles comme beaucoup plus exigeantes qu'ils ne s'y attendaient au départ. Ils considèrent qu'il leur manque des indications claires permettant de les guider adéquatement dans l'exercice de leur rôle. Autant la mère que le père peuvent done bénéficier de mesures visant à améliorer les habiletes parentales.

L'importance de s'adresser autant à la mère qu'au père ou au conjoint est cruciale dans le cas des familles négligentes puisque ce sont autant les comportements individuels des deux parents que la dynamique d'ensemble de la famille qui font problème. 


\section{PROBLÉMATIQUE}

Même si le nombre de recherches sur la négligence est réduit, cerrains constats ont néanmoins été dégagés. L'urgence d'intervenir auprès de ces familles afin d'éviter les effets néfastes de la négligence sur les enfants est reconnue par tous ceux qui sont d'une manière ou d'une autre confrontés avec ces réalités. Mais comment intervenir de façon efficace, voila qui est bien moins clair. Une des rajsons de cet état de fait est certes le peu d'expériences systématiques et de recherches sur l'intervention avec des familles négligentes. Mais une autre raison est la difficulté même d'enclencher un processus d'intervention ainsi que d'éveiller et de maintenir l'intérêt des parents négligents dans un processus d'intervention. Dans ce cadre, l'analyse du processus même de l'intervention revêt un intérêt particulier. $C^{\prime}$ 'est cette dernière dimension qui retient ici notre attention.

L'objectif spécifique de cette évaluation de processus est donc d'analyser la participation des parents négligents à un groupe destiné à améliorer les habiletés parentales et la capacité de résoudre des problèmes de la vie quotidienne, Compte tenu des besoins et des caractéristique spécifiques des parents négligents, il s'agit done de se demander:

- Dans quelle mesure les parents négligents participent-ils à ce type d'activité? Quel est leur taux de présence? Quel est leur degré de participation?

- Quel est le niveau de prise de conscience de leurs problèmes manifesté par les parents négligents au sein du groupe?

- Quelles sont les principales préoccupations manifestées par ces parents?

- Quels sont, selon la perception des animateurs, les événements les plus significatifs lors des rencontres?

- Quelles sont les impacts de l'activité, selon la perception des parents?

\section{MÉTHODOLOGIE}

\section{Échantillon}

L'échantillon est constitué de parents négligents présentant ou non des comportements violents et ayant au moins un jeune enfant âgé entre zéro et six uns. Il s'agit de familles qui, suite à un signalement, ont été retenues par le CPEJ-MBF en conformité avec la loi de la protection de la jeunesse, comme présentant de la négligence ou de la négligence et de la violence physique envers au moins un de leurs enfants. Trente-cinq familles ont accepté au départ de participer au programme. Sept hommes n'ont pas voulu s'impliquer dans la démarche de groupe.

L'échantillon de départ est de 50 adultes dont 33 femmes et 17 hommes. Les personnes ayant participé à tout le programme, c'est-à-dire celles ayant continuté jusqu'à la fin des activités telles que prévues sont au nombre de 38 , dont 29 femmes et 9 hommes (sous-groupe Participant). Douze personnes, dont quatre femmes et huit hommes, ont quitté le programme à divers moments au cours de la première moitié de son déroulement (sous-groupe Abandon).

Les familles avaient été sélectionnées à partir d'un signalement touchant un enfant mais nous trouvons, à l'intérieur de ces familles un nombre élevé d'enfants 
(105 enfants). En effet, presque la moitié de l'échantillon (47\%) est constitué de familles avec trois enfants et plus. Par ailleurs, il est important de souligner ici que 13 plupart des familles $(73 \%)$ avaient été portées à l'attention de la CPEJ à plusieurs reprises pour l'un ou l'autre enfant. Un total de 150 signalements retenus à la CPE pendant les cinq années précédant l'expérience ont étế répertoriés pour les 105 enfants. Il s'agit done dans la majorité des cas de récidives et donc de familles très dysfonctionnelles et présentant une problématique achronique. Il s'agit également de familles très défavorisées sur le plan éducationnel (huit ans de scolarité en moyenne autant pour les femmes que pour les bommes, et $53 \%$ des mères et des pères ou conjoints ayant redoublé des années ou fréquenté des classes spéciales) et socio-économique ( $81 \%$ vivant de la sécurité sociale depuis longtemps, $9 \%$ de l'assurance chômage, et $9 \%$ d'un emploi à bas revenu). Une variété de structures familiales a été observée dans l'échantillon. Les familles monoparentales sont surreprésentées (40\%). Seulement $29 \%$ sont des familles biparentales d'origine, $23 \%$ sont des familles reconstituées (six mois et plus de vie en commun), et $9 \%$ sont des unions récentes.

\section{L'ACTIVITÉ DE GROUPE DESTINÉE AU aPARENT EN TANT QUE PARENT*}

Le programme proposé aux parents combine des dimensions de développement de la capacité du parent de résoudre des problèmes, de développement d'habiletés parentales et d'information sur des questions variées. II s'agit d'une activité de groupe des mères et des pères ou conjoints animée par un professionnel. Quelques rencontres incluent les enfants. Les activités se déroulent soit avec l'ensemble du groupe, soit en sous-groupes.

Le programme s'articule autour de quatre grands objectifs spécifiques:

(1) Développer chez le parent l'habileté à résoudre des problèmes et à planifier ses activités quotidiennes.

(2) Améliorer la relation du parent avec son enfant et lui apprendre à faire l'autocritique de son propre comportement.

(3) Améliorer la connaissance de son enfant, de ses besoins émotionnels et de ses capacités, apprendre à voir son enfant de manière plus positive. Apprendre à mieux répondre aux besoins physiques et quotidiens de l'enfant, à faciliter le développement de certaines habiletés chez son enfant et à communiquer avec son enfant à travers le jeu.

(4) Acquérir des informations sur les ressources communautaires et apprendre à les utiliser.

Outre ces objectifs spécifiques, l'activité avait comme but de donner à ces parents l'occasion de sortir de leur isolement, d'échanger avec d'autres parents, et de vivre une expérience sociale nouvelle. On voulait également essayer d'augmenter la quantité et la qualité de l'implication des pères ou conjoints auprès de l'enfant en particulier, et de la famille en général.

Le programme est constitué d'un total de 20 séances. L'animateur doit suivre les objectifs fixés mais il doit tenter de le faire à partir des problèmes rapportés par les parents eux-mêmes. Dans les premières rencontres, l'animateur doit surtout 
laisser les parents exprimer leur ambivalence envers leur enfant et leur ròle pa. rental et il doit montrer une acceptation de ces sentiments. Par contre, l'animateur
montrera clairement que certains actes ne peuvent pas être acceptés.

Dans la partie d'entrainement à la résolution de problèmes, une série de problèmes de base servent à l'entrainement. Ensuite, ce sont des problèmes proposés par certains parents qui doivent être résolus par les autres parents. Les participants proposent diverses solutions qui sont analysées ensuite en groupe afin de voir les conséquences positives et négatives de chaque solution. On tente ensuite de dégager quelle est la meilleure. Si nécessaire, l'animateur donne un feedback. Plus tard, on travaille à améliorer les solutions déjà trouvées.

Sans perdre pour autant de vue les objectifs spécifiques, l'animateur doit tenir compte des besoins exprimés par les parents et se montrer accueillant envers leurs préoccupations, même si elles ne correspondent pas aux objectifs spécifiques.

\section{IMPLANTATION DE L'INTERVENTION ET DESCRIPTION DE L'INSTRUMENT D'ÉVALUATION DU PROCESSUS}

La possibilité de participer au programme PAPFC fut offerte dans quatre points de services différents du CPEJ-MBF desservant une population urbaine et une population rurale et semi-rurale (Trois-Rivières, Shawinigan, Drummondville, et Victoriaville) comme partie des mesures volontaires proposées aux parents des jeunes enfants retenus en tant que victimes de négligence à l'automne 1992. Les intervenants de ces quatre succursales de la CPEJ-MBF ont offert aux parents de chaque cas nouvellement retenu pour négligence deux possibilités différentes à titre de mesures volontaires: la participation à l'ensemble du programme PAPFC offert par le Groupe de recherche en développement de l'enfant et de la famille en collaboration avec la CPEJ-MBF ou les mesures proposếes par l'intervenant dans le cadre des modes d'intervention habituels de la CPEJ. Des informations complètes relatives aux objectifs, au calendrier et aux services offerts leur ont été fournies. Tous les parents ayant reçu l'offre ont choisi de participer au PAPFC. Une seule famille participa sous ordonnance du juge, L'échantillon est donc très représentatif de la population retenue pour négligence majeure dans le services de protection.

Les parents ont été également informés des buts de la recherche et leur consentement à participer leur a été demandé. La confidentialité de tous les renseignements recueillis aux fins de ta recherche a été garantic aux participants ainsi que leur total liberté d'expression au sein du groupe.

Quatre groupes furent done formés selon le lieu de résidence de la famille. Autant les mères que les pères ou les conjoints et conjointes furent invités à l'activité de groupe.

Chaque groupe fut animé par un psychologue avec expérience dans l'animation de groupes de parents et dans le travail clinique. Mais aucun d'entre eux n'avait travaillé avec des groupes de parents négligents ou n'avait de liens avec le CPEJ. Trois séances de discussion des quatre animateurs avec la chercheure principale furent tenues afin de partager les orientations à donner à l'activité et à lanimation des groupes. Bien que devant suivre les objectifs spécifiques de l'activité tels que décrits auparavant, les animateurs reçurent la consigne d'être très 
souples, de surtout encourager les parents à exprimer leurs préoccupations et de tenter de répondre le plus possible aux besoins exprimés par les parents. En outre, les animateurs ont participé tout au long de l'expérience aux rencontres de l'équipe elinique qui effectuait le suivi individuel de chaque famille. Ils étaient done informés de la situation de chaque cas et de leur problématique spécifique.

Une grille d'observation (Palacio-Quintin \& Desaulniers, 1992) devant être retaplie par chaque animateur à la fin de chaque séance a été utilisée afin de systématiser le recueil des observations des animateurs sur le fonctionnement du groupe et de chaque participant au sein du groupe. Une première section de la grille permet d'identifier trois questions à propos de chaque participant: (a) la présence ou l'absence de chaque membre à la séance en question; (b) l'appréciation de l'animateur sur le degré de participation de chacun aux échanges dans le groupe. II s'agit ici de savoir dans quelle mesure, au-delà de la présence physique, chaque personne participe plus ou moins activement dans les échanges, les décisions, et les projets du groupe: (c) l'appréciation de l'animateur sur la prise de conscience de ses problèmes par chaque parent. Il $\mathrm{s}^{\prime}$ agit ici d'un jugement clinique émis par lanimateur à partir de l'expression verbale des participants.

Pour les questions b et $c$, les animateurs devaient répondre sur une échelle Likert en cinq points allant de la participation ou la prise de conscience nulle (1) à très grande (5). Dans la question $\mathrm{c}$, il $\mathrm{y}$ avait un sixième point à l'échelle: uimpossible d'appréciers. En effet, dans des circonstances où un participant s'exprime peu, l'animateur ne peut pas préjuger du niveau de conscience que le participant possède, puisqu'il s'agit d'un jugement clinique sur le contenu de ce que le participant exprime et non d'une évaluation de la quantité d'expression verbale.

Une deuxième partie permet aux animateurs de rapporter des informations sur les principaux thèmes abordés dans le groupe et sur ce qui, selon eux, intéresse le plus l'ensemble des parents. Enfin, une troisième partie permet aux animateurs de rapporter les elements qui attirent leur attention et leur apparaissent significatifs, compte tenu de leur expérience clinique et leur expérience d'animation des groupes de parents.

Une rencontre des quatre animateurs avec les auteures a servi à donner les consignes relatives à la manière de remplir la grille en s'assurant ainsi de la plus grande uniformité possible.

Le programme s'est déroulé selon la planification, c'est-à-dire qu'il y a cu 20 rencontres pour chaque groupe divisé en deux grands blocs, le premier ayant eu lieu entre février et mai 1993 et le deuxième bloc entre les mois de septembre et décembre de la même année. Aucun effort n'a été épargné pour faciliter la participation des parents à l'activité de groupe. Une garderie sur place et le transport étaient offerts, de manière à ce qu'il n'y ait pas d'empêchements matériels à la participation. Du soutien psychologique fut aussi offert, comme téléphoner à certains participants deux heures avant l'activité pour qu'ils n'oublient pas de s'y rendre. Une rencontre préalable préparatoire pour le début du programme PAPFC a permis aux participants de rencontrer une première fois l'animateur et les autres Participants et de connaitre les objectifs spécifiques à l'activité du groupe «Le parent en tant que parent. 
Les animateurs ont rempli la grille après chaque séance. Nous avons done un total de 80 observations (20 observations par quatre groupes). Le fait de compter sur quatre groupes fonctionnant de façon indépendante et sur quatre animateurs qui font les observations et commentaires nous permet de généraliser ces données cliniques.

A la fin du programme, une rencontre de groupe fut tenue avec les parents de chaque point de services afin de recueillir leur perception sur l'ensemble du programme et sur l'activité de groupe en particulier. Un membre de l'équípe anima la rencontre en laissant les parents s'exprimer et discuter librement.

\section{RÉSULTATS}

Les analyses sont présentées en quatre parties. Les données quantitatives (première partie de la grille) sur le taux de présence, le degré de participation aux échanges dans le groupe et le degré de prise de conscience des problèmes sont abordées en premier lieu. Les trois autres parties rapportent les analyses de contenu des données qualitatives (deuxième partie de la grille), D'abord, nous retenons les principaux thèmes abordés et ce qui a semblé intéresser te plus l'ensemble des parents. Ensuite, nous regardons quelles sont les questions

\section{TABLEAU 1}

Taux de présence et degré de participation des parents négligents à l'activité de groupe et degré de prise de conscience sur leurs problèmes

\begin{tabular}{lrcrc} 
& Groupe Abandon & \multicolumn{2}{c}{ Groupe Participant } \\
& \multicolumn{2}{c}{ Taux de présence à l'activité de groupe } \\
Femmes & N & $\%$ de présence & N & $\%$ de présence \\
Hommes & 4 & $45 \%$ & 29 & $79 \%$ \\
Total & 8 & $41 \%$ & 9 & $67 \%$ \\
& 12 & $42 \%$ & 38 & $76 \%$
\end{tabular}

Femmes

Hommes

Total

Degré de participation aux échanges à l'intérieur du groupe

$\begin{array}{lcccc}\text { Femmes } & \text { N } & \text { Moy. (écart t.) } & \text { N } & \text { Moy. (écart t.) } \\ \text { Hommes } & 4 & 1,70(0,68) & 29 & 2,73(0,76) \\ \text { Total } & 5^{*} & 1,96(1,20) & 8^{*} & 2,80(0,76) \\ & 12 & 1,84(0,96) & 38 & 2,76(0,75)\end{array}$

\footnotetext{
- Trois membres du groupe Abandon et un membre du groupe Participant ne sont pas inclus jei puisque les animateurs ont coté -impossible d'apprécier. dans toutes les séances.

Groupe Abandon: Parents ayant abandonné le programme pendant la première moitié.

Groupe Participant: Parcats ayant participé tout au long du programme.
} 
apparues comme particulièrement significatives aux yeux des animateurs. Pour terminer, nous rapportons une analyse des propos tenus par les parents eux-mémes fors de la rencontre d'évaluation finale.

\section{A. Présence, participation, et prise de conscience}

Un sous-groupe de parents représentant $24 \%$ de l'échantillon initial a abandonné le programme pendant son déroulement. Le plus grand nombre d'abandons se trouvent chez les hommes $(47 \%)$ tandis que seulement $12 \%$ des femmes ont abandonné. Les informations disponibles pour ces personnes pendant qu'elles participaient sont intéressantes à mettre en parallèle avec celles touchant les personnes qui ont persisté jusqu'à la fin des activités. Nous présentons donc les observations sur ce groupe (groupe Abandon) aussi bien que celles du groupe ayant participé tout au long du programme (groupe Participant). Dans le cas du groupe Abandon, le taux de présence est calculé par rapport à la période où ces personnes faisaient partie du programme (nombre de séances de groupe ayant eu lieu jusqu'à l'abandon). Les résultats sont rapportés dans le tableau 1, autant pour l'ensemble de parents que pour les femmes et les hommes séparément.

Le pourcentage moyen de présence pour le total de l'échantillon est de $68 \%$ et les femmes se sont présentées aux trois-quarts des rencontres $(75 \%)$ alors que les hommes ne se sont présentés qu'à $55 \%$ des rencontres. Le taux de présence est différent quand nous considérons séparément les sous-groupes Abandon et Participant. Les parents qui ont participé à l'ensemble du programme ont été présents aux trois quarts des rencontres alors que ceux qui ont abandonné avant la fin ont. pendant qu'ils faisaient encore partie du programme, un taux de présence de $42 \%$. Pour le type de clientèle de notre étude. le taux de présence dans la première moitié de l'activité de groupe apparait done comme un indice clair du véritable désir d'entrer dans une démarche et de la mener à bien. On peut observer également que les hommes ont plus tendance que les femmes à abandonner ce type de service. En effet, dans notre clientèle totale de 17 hommes, huit ont quitté le programme alors que quatre femmes seulement sur 33 l'ont fait. Par contre, il n'y a pas pour ce groupe de différence entre le pourcentage de présence des femmes $(45 \%)$ et des hommes ( $41 \%)$ pendant qu'ils étaient dans le programme. Pour le groupe ayant participé tout au long du programme, nous constatons par contre une présence plus assidue des femmes (12\% de plus que les hommes).

En ce quí à trait à la participation active au sein đu groupe, l'évaluation faite par les animateurs montre un niveau moyen d'engagement dans les échanges de la part de l'ensemble de l'échantillon $(\bar{X}=2,76$. écart type 0,64$)$. La courbe de répartition entre les cinq cotes est régulière, avec peu de cas extrêmes. Les parents ayant abandonné le programme participaient moins dans le groupe quand ils étaient présents. Cette différence est plus accentuée chez les femmes. Le degré de participation parmi ceux qui ont participé à tout le programme ne semble pas varier beaucoup selon le sexe, mème sì nous observons une très légère tendance des femmes a s'impliquer un peu plus activement que les hommes.

Le niveau de prise de conscience de leurs problèmes que les parents de l'échantillon total manifestent au sein du groupe est considéré par les animateurs comme moyen faible $(\bar{X}=2,57$, écart type $=0,86)$. Cette appréciation a peu varié, dans le sens d'une baisse, entre la première et la deuxième partie de la démarche. 
En effet, le score moyen au premier bloc de séances $(\bar{X}=2,81)$ et le score moyen au deuxième bloc de séances $(\overline{\mathrm{X}}=2.61) \mathrm{n}$ 'indiquent pas de changements importants à travers le temps pour l'ensemble du groupe. Il ne semble pas non plus y avoir eu des changements intraindividuels marqués puisque l'analyse de l'évolution de chaque participant nous indique qu'il $n^{\prime} y$ a aucune variation (passage d'une catégorie à l'autre, éest-à-dire + ou -1 dans le score moyen) chez $66 \%$ des participants, qu'il y a une augmentation de la prise de conscience chez $14 \%$ des participants tandis que les autres $20 \%$ sont considérés par les animateurs comme manifestant un niveau de conscience inférieur pendant la deuxième partie.

En fait, ceci n'est pas étonnant. II faut se rappeler qu'il s'agit de parents négligents *chroniques». qui ont comme caractéristique un manque de conscience de leurs responsabilités et qui semblent également avoir peu de conscience de leurs propres problèmes. Ils ont plutôt tendance à attribuer leurs problèmes à des facteurs externes et à avoir le sentiment qu'ils ne peuvent pas contrôler les événements, ni leurs relations avec les autres. Mais les données permettent de voir qu'il y a une différence dans le degré de prise de conscience manifesté chez les deux sous-groupes, et ce tant pour les femmes que pour les hommes.

Une différence est perceptible au niveau de la clientèle qui s'est retirée du programme. Nous voyons ici un plus faible niveau de conscience de leur problématique, ce qui implique un faible degré de responsabilisation et par conséquent, peu de motivation à poursuivre une activité dans laquelle ils ne se sentent pas concernés.

Dans la elientèle ayant persisté tout au long du programme, le niveau de conscience manifesté par les hommes et par les femmes est très semblable, avec une très faible supériorité du score des femmes. Par contre, on peut observer que le score moyen des hommes est légèrement supérieur à celui des femmes dans le cas des participants qui ont abandonné le programme. C'est le cas du moins chez ceux pour lesquels les animateurs ont été capables de faire l'évaluation. En effer. les animateurs ont considéré, pour trois des huit hommes ayant suivi le programme, qu'il ne leur était pas possible de se prononcer sur leur niveau de conscience et ce, à aucune des séances du groupe. La même situation s'est présentée avec un des quatre hommes ayant abandonné le programme. Il n'y a aucun cas de femme où l'animateur a jugé qu'il ne pouvait pas se prononcer. II semble done qu'il y ait moins d'expression personnelle de la part des hommes au sein du groupe et qu'ils ont tendance à masquer davantage leurs pensées et leur sentiments.

\section{B. Les thèmes traités et les intérêts manifestés}

Les thèmes traités dans les quatre groupes sont multiples mais il se dégage des rapports des quatre animateurs des constantes dans le type de thèmes retenus ainsi qu'une stabilité au niveau de la progression des thèmes abordés spontanément par les parents, à mesure que les rencontres se succédaient.

Les thèmes abordés touchent difrérents aspects qu'il est possible de classer en quatre grandes catégories de préoccupations: celles touchant les enfants: celles qui ont trait aux relations et à l'organisation familiales; celles par rapport à soi-méme en tant qu'individu, et des préoccupations d'ordre matériel. 
Par rapport à la préoccupation quant aux enfants, on voit apparaître des questions et des échanges d'informations sur les grandes règles de développement des enfants de zéro à six ans mais surtout sur des questions très simples de la vie quotidienne: depuis "comment utiliser un thermomètren, en passant par nquoi faire quand un enfant pleure ou eque doit-on lui fournir comme alimentation pour qu'il se développe normalemente, jusqu'à chercher à savoir s'il west normal que l'enfant se touche les parties sexuelles à 5 anss ou si l'on adoit parler à son jeune enfant.. L'apparition de ces thèmes allait de soi en fonction des objectifs spécifiques définis pour l'activité, objectifs qui ont été véhiculés clairement par les animateurs lors de la rencontre préparatoire à l'entrée au programme PAPFC. On peut s'attendre à voir surgir ces thématiques dans n'importe quel groupe de parents, mais ce qui est peut être particulier est l'apparition de certains questionnements sur des choses extrèmement simples qui se transmettent normalement au sein de la famille et qui montrent combien ces parents sont désarmés pour répondre aux besoins élémentaires de leurs jeunes enfants.

L'autre préoccupation très présente est celle des relations familiales: relations mère-père, parents-enfants, et enfants-enfants. Le problème de la violence avec et entre les enfants a surgi dans chacun des groupes et tous les groupes se sont penchés sur les thématiques des punitions, des règles et des normes, du respect mutuel, et par conséquent sur les moyens à prendre pour que l'organisation de la vie familiale soit mieux adaptée. Ici encore, on peut observer que les parents négligents possèdent peu de moyens pour intervenir ou communiquer aveo leurs enfants. Ils connaissent surtout des méthodes punitives pour contrôler leurs enfants. Les formules qu'ils disent utiliser pour imposer le respect des normes ou tenter de communiquer avec leurs enfants sont toujours négatives. II apparait que la plupart de ces parents semblent ignorer toute autre forme d'intervention pour favoriser l'acquisition de nouveaux apprentissages chez leurs enfants et des interactions plus positives. En outre, quand ils arrivent à conceptualiser une autre forme d'approche plus positive, ils espèrent des progrès immédiats. La dimension de la persévérance dans la tâche d'éducation leur fait cruellement défaut tant par leurs propres problèmes affectifs qui les amènent à chercher un délai court entre l'émergence d'un besoin et la satisfaction de celui-ci, que par la connaissance réduite des besoins et des différents mécanismes d'apprentissage chez le jeune enfant, En effet, laspect communication parents-enfants à travers le jeu ou par l'intérễt porté aux activités de leur enfant ou par le toucher affectueux semble être très réduit.

Une troisième grande thématique relève plus des parents en tant qu'individus. Les participants exprimaient souvent leur besoins en tant que conjointe ou conjoint, en tant que mère ou père, ou simplement en tant que femme ou homme. On retrouve beaucoup de préoccupations concernant leur besoin d'être aimés et respectés par leur conjoint(e) et par leurs enfants. D'autres besoins d'affiliation entre les différents membres de lì famille sont également identifiés comme le besoin de complicité, d'avoir sa place dans la vie familiale, etc.

Enfin, une quatrième catégorie de thèmes a trait aux préoccupations matérielles des parents. À l'occasion, on parle de la gestion du budget, de recettes de cuisine simples et peu coûteuses, de recherche d'emploi, etc. 
Il a été également constaté une progression des thèmes soulevés à mesure que les rencontres se succédaient. Alors que dans les premières rencontres, les participants cherchaient surtout à se connaitre et à définir leurs sujets d'intếrêt par rapport aux habiletés parentales et leurs moyens pour résoudre les problèmes qui se posent avec leurs enfants, on observe par la suite qu'ils parlent davantage de leur vécu immédiat. Dans les dernières rencontres par contre, les activités prévues avec les enfants ou les activités sociales prennent de plus en plus d'importance.

II ressort clairement des observations des animateurs que c'est l'activité de groupe en soi qui a attiré davantage l'intérêt des participants plutôt que les objectifs spécifiques sur les habiletés parentales. La plupart des parents désirent surtout parler de leur vécu et écouter les autres parler du leur. Ils veulent faire des liens avec leurs propres expériences de parents mais également avec leur propre enfance. C'est également l'aspect social des rencontres, se parler en petit groupe, s'échanger des recettes, des informations en dehors de la tâche, créer de nouveatux liens, s'entraider. Bref, c'est leur besoin d'établir des contacts, de sortir de leur isolement, et de partager leurs difficultés avec d'autres qui semble être leur besoin principal.

\section{Les aspects le plus significatifs selon les animateurs}

Cette information colligée auprès des quatre animateurs peut se classer en trois grandes catégories comportant chacune un pôle positif et un pôle négatif.

Un certain nombre de comportements individuels a attiré d'abord l'attention des animateurs. Les animateurs observent des attitudes marquées de fuite, de négation, de déresponsabilisation, et de méfiance non seulement face à l'animateur mais également face aux autres membres du groupe. Les animateurs sont également étonnés par "l'impatience" qui caractérise les participants, c'est-à-dire leur délais temporels très courts. En effet, plusieurs participants manifestent l'attente qu'avec un petit geste, on pourra changer une situation complexe et qui perdure depuis longtemps. Du côté positif, les animateurs remarquent chez les participants de l'initiative, des prise de conscience (insight) parfoís étonnantes (mais pas régulières), la reconnaissance de leurs responsabilités, et des démarches impliquant une demande d'aide. Pour comprendre l'importance de ce dernier élément, il faut se rappeler que cette clientẻle n'a pas d'elle-même demandé de l'aide. Une diminution de la méfiance et une augmentation marquée de la confiance envers les animateurs est également constatée à mesure que l'activité de groupe se déroule.

D'autres observations visent la dynamique des groupes. Du côté négatif, les animateurs relèvent des mouvements de résistance et des comportements agressifs dans les groupes, ainsi que des conflits importants provoqués par la médisance. Mais d'autre part, les animateurs relèvent des attitudes de solidarité. la manifestation de sympathie face aux problèmes des autres, la manifestation d'initiative pour favoriser la résolution de problèmes dans le groupe et pour développer des activités sociales. Enfin certains liens se sont créés entre les participants, liens qui se sont prolongés au-delà de l'activité de groupe.

Enfin, les animateurs sont frappés par les situations difficiles et la quantité d'événements troublants auxquels sont ou ont été exposés les participants. On peut voir que plusieurs ont vécu des situations d'abus sexuel, de violence, de négli- 
gence, de placement, de deuil, etc., et qu'ils vivent en tant que parents des placements de leurs enfants. Ils vivent cette situation de manière très douloureuse, même s'ils n'arrivent pas à faire face aux besoins des enfants quand ces derniers sont avec eux. Le *On les aime quand même .... dit par une mère résume bien le drame. En tant qu'adultes, ils vivent également beaucoup de séparations, d'états dépressifs, de troubles de consommation d'alcool et de drogues, etc. Des difficultés cognitives chez un bon nombre de participants sont également observées par les animateurs et confirmées par les tests psychométriques (le test des Matrices progressives de Raven) utilisés dans le cadre global de la recherche. Ceci demande une double adaptation de la part des animateurs dans la manière de conduire l'activité de groupe.

En fait, on peut constater ici que les parents négligents sont exposés à un nombre d'événements stressants qui semble bien plus grand que la population générale des parents. Bref, les animateurs constatent qu'il est impossible de détacher les problèmes parentaux de l'ensemble des difficultés que vivent ces parents.

\section{La perception de la clientèle elle-même sur l'activité de groupe}

Lors de la rencontre d'évaluation de groupe, l'ensemble de participants affirment avoir beaucoup apprécié l'activité de groupe et pour plusieurs, l'activité de groupe a été sélectionnée comme le service le plus apprécié de tout notre programme PAPFC. Ils ont également exprimé qu'ils regrettaient que l'activité soit terminée et qu'ils auraient désiré continuer.

L'ensemble des participants disent avoir aimé les activités de groupe parce que ces dernières leur ont permis de créer des liens de confiance et d'amitié et de se soutenir mutuellement. L'ensemble des participants rapportent s'être sentis acceptés et respectés par les autres. Ceci est particulièrement important pour des personnes qui manifestent une faible estime de soi et qui se sentent souvent rejetées et mal jugées socialement.

Bon nombre de participants considèrent avoir, grâce à l'activité de groupe, augmenté leur confiance en soi eu égard à leur capacité à entrer en relation avec les autres. Les participants se sont découverts des capacités à mieux comprendre les autres et à s'ouvrir plus aux différences et pour plusicurs le groupe a été un agent de changement dans les comportements à adopter dans une activité sociale.

Pour plusieurs, le groupe a été aussi perçu comme un véhicule très efficace au plan de l'éducation des enfants, de la communication avec eux, et des solutions apportées par les animateurs ou par d'autres participants, pour résoudre des situations problématiques.

Enfin, la plupart des participants ont indiqué clairement que les groupes avaient été un véhicule de changement très significatif tant au plan de leurs habiletés parentales qu'au niveau de leur évolution personnelle. En effet, certains rapportent avoir acquis beaucoup de nouvelles connaissances, d'autres affirment qu'ils ont pu développer des compétences personnelles qui les ont aidés à appliquer ce qu'ils savent devoir faire.

Ces remarques faites par les parents participants eux-mêmes laissent croire que l'activité de groupe telle que menée semble avoir répondu à un besoin impor- 


\section{REVUE CANADIENNE DE SANTEE MENTALE COMMUNAUTAIRE}

tant chez eux, besoin auquel ils trouvent peu souvent réponse. En effet, ces remarques positives sont particulièrement significatives puisque ces mêmes parents négligents ont une tendance générale à porter des jugements négatifs et à se montrer méfiants envers les intervenants.

\section{CONCLUSIONS}

L'analyse des données sur le taux de présence et de participation des parents et le degré de prise de conscience de leurs problèmes permet d'abord de distinguer clairement les parents qui ont abandonné la démarche de ceux qui ont persisté. En effet, les 12 personnes ayant quitté le programme s'absentaient très souvent, dès le départ. Quand elles étaient présentes, elles s'impliquaient moins que les autres dans les échanges du groupe et manifestaient un degré de conscience de leurs difficultés qualifié de presque nul par les animateurs. Par exemple, il est arrivé, au moment où l'on discutait dans le groupe sur ce que les parents estimaient eux-mêmes être des raison pour les amener à entreprendre une telle démarche, que l'un d'entre eux dise ne pas savoir pourquoi il était là. Or, depuis le signalement jusqu'à l'entrée des parents dans le groupe, toute une démarche avait été réalisée par les intervenants de ta CPEJ-MBF avec chacun des parents pour leur expliquer les failles dans leur manière d'assumer leur rôle parental, failles qui étaient inacceptables et qui causaient grand tort à leur enfant, et qui exigeaient que des mesures soient prises pour modifier la situation. Ces éléments nous permettent de voir que le comportement des parents pendant les 10 premières rencontres permet de prédire leur capacité de profiter de l'activité et d'intégrer la démarche. Un seuil minimal de conscience de ses propres besoins semble donc essenticl pour qu'un parent en difficulté puisse profiter d'une démarche de groupe de ce type.

On constate également que les hommes ont été nombreux à abandonner le groupe. En effet, $47 \%$ des hommes ont quitté la démarche alors que seulement $12 \%$ des femmes l'ont fait. Les hommes qui sont demeurés tout au long du programme se sont ausst absentés plus fréquemment que les femmes (12\% d'absences de plus), mais, lorsqu'ils étaient présents, ils participaient presque autant que les femmes et manifestaient le même niveau qu'elles de prise de conscience de leurs problèmes.

L'ensemble des participants a été présent aux trois quarts des séances, taux de présence élevé pour une clientèle qui est connue comme udécrocheusè de toutes les démarches qui lui sont proposées. Ce taux est beaucoup plus élevé que celui đé $50 \%$ rapporté par Landsman et al. (1992) dans une expérience de groupe qui. rappelons-le, était peu structurée. L'expérience de groupe offerte dans notre programme aux parents négligents a réussi davantage à enclencher leur intérét pour s'inscrire dans une démarche de changement. Le niveau de participation au sein du groupe, qualifić globalement de moyen par les animateurs, laisse croire que malgré leurs difficultés ces parents peuvent bien participer et done tirer profít d'une démarche de groupe quand, tout en étant bien encadrés, ils se sentent écoutés et compris.

Les autres éléments analysés nous permettent de voir que c'est surtout l'activité de groupe en soi qui a pris le plus d'importance et qui a intéressé le plus ces parents. En effet, tout en abordant des questions par rapport à leurs enfants ot 
en reconnaissant que l'activitế de groupe les a aidés à acquérir des habiletés parentales, les parents expriment surtout l'importance que le groupe a eu pour eux du point de vue affectif et social. Ils disent se sentir acceptés, plus confiants dans leurs relations avec les autres, plus à l'aise dans les échanges sociaux et avoir trouvé du soutien mutuel. Pour certains d'ailleurs, l'activité de groupe et la confiance développée face à l'animateur ont été le déclencheur qui leur a permis de faire d'eux-mêmes une démarche de demande d'aide psychologique.

L'analyse des thématiques abordées spontanément par les parents permet de voir que les questions qui ont trait aux enfants dérivaient souvent et rapidement vers des questions sur les relations avec le conjoint et avec l'ensemble de la famille et sur leurs propres besoins en tant que personne. Les conclusions des animateurs voulant qu'il soit impossible de détacher les problèmes parentaux de l'ensemble des difficultés que vivent ces parents coīncident bien avec ce que les parents ont exprimé eux-mêmes indirectement. Il apparait done qu'il peut être illusoire de vouloir, dans les cas des parents négligents chroniques, agir directement sur les comportements parentaux. Traiter les problèmes parentaux peut être inefficace si V'on ne répond pas simultanément aux multiples préoccupations personnelles et relationnelles des parents négligents. Il s'avère done nécessaire de combiner dans lactivité de groupe le travail sur les habiletés parentales avec un approche thérapeutique qui tient compte des problèmes et des besoins des parents euxmẻmes.

Les parents négligents disent avoir beaucoup profité de l'activité de groupe. À notre avis, cela n'a été possible que grâce à l'orientation particulière donnéc à l'activité et au rôle très important de médiateurs joué par les animatcurs. En effet, au cours du premier bloc de rencontres, beaucoup de conflits, d'agressivité marquée, et de rejet de certains membres vis-à-vis d'autres se sont manifestés. Sans la médiation de l'animateur, les situations auraient pu rapidement dégénérer en conflit généralisé et en abandon de l'activité de groupe par plusieurs participants. Ceci aurait constitué un échec de plus dans leur vécu d'interaction avec les autres, qui n'aurait fait que confirmer leur faible estime d'eux-mémes. Nous voyons done l'importance de pouvoir mettre à la portée de ces parents, qui vivent tant de problèmes relationnels, la possibilité de participer à des démarches collectives orientées et menées par des professionnels compétents.

L'approche de groupe est une forme d'intervention prometteuse pour oeuvrer auprès des parents négligents. II y aurait intérêt à envisager son introduction dans le système d'intervention de la CPE, Mais il faut se garder de croire que n'importe quelle forme d'activité de groupe peut être utile. L'intervention en groupe doit considérer autant les problèmes parentaux que personnels des parents négligents dans une perspective thérapeutique. En outre, il est important de souligner que le rôle de l'animateur de ce groupe est incompatible avec le rôle de l'intervenant de la CPEs (la «personne autoriséeu), personne qui tout en essayant d'aider les parents a un rôle de contrôle social et légal. L'animation du groupe doit donc être assumée par un autre professionnel que celui qui est te responsable du suivi des cas. Des activités de groupe de type communautaire ne peuvent à notre avis, dans un premier temps, répondre adéquatement aux besoins des parents négligents. Par Contre, une fois franchie une première phase où ces personnes développent un thinimum de confiance en soi et dans les autres, où elles se sentent un peu plus 
adéquates en société, les activités en groupe communautaire ou d'entraide peuvent alors constituer un apport important à leur développement. En fait, ces activités communautaires peuvent probablement être indispensables dans une deuxième phase du processus de rétablissement des parents négligents, phase où ces personnes doivent exercer davantage des interactions sociales en dehors d'un milieu très protégé comme l'a été le groupe constitué pour l'expérience.

\title{
NOTES
}

1. À partir de l'entrée de la clientèle à notre programme PAPFC, ancun placement a'a été effectué, mais certaines familles avaient des enfants placés, suite à des signalements retenus antérieurement envers un autre de leurs enfants.

\begin{abstract}
This document reports a process evaluation of a group experience with neglectful parents. Obviously, neglectul parents have difficulty assuming parental responsibilties, but they are also characterized by poor living situations, isolation, and poor integration in their community. A group experience was undertaken with 35 families ( 33 women and 17 men) identified by the protection agency (CPEJ) as neglecting their young children. The parents were divided into four groups, each led by a professional. The authors analyze the group process and its contribution to dealing with parents who have very specific needs.
\end{abstract}

\section{RÉFÉRENCES}

Aderman, J. \& Russell. T. (1990). A constructivist approach to working with abusive and neglectful parents. Family Systems Medicine, 8, 241-250.

American Psychiatric Association. (1994). Diagnostic and statistical manual of mental disorders (3e ed.). Washington. DC: Auteur.

Azar. S.T. (1984). An evaluation of the effectiveness of cognitive-behavioral versus insightoriented mothers' groups with child maltreatment cases. Thèse de doctorat inédite, University of Rochester.

Barth, R.P. (1985). Beating the blues: Cognitive-behavioral treatment for depression in childmaltreating young mothers. Clinical Social Work Journal, 13(4), 317-328.

Bernal, M.E., \& North, J.A. (1978). A survey of parents' training manuals, Jourtial of Applied Behavior Analysis, 11, 533-544.

Chamberland, C.. Bouchard, C., \& Beaudry, J. (1986). Conduites abusives et négligenles envers les enfants: réalités canadiennes et américaines. Revue canadienue des sciences du comportement, $18,391-412$.

Cohn, A.H. (1979). Essential elements of successful child abuse and neglect treatment. Child Abuse and Neglect, 3, 491-496.

Crittenden, P. (1988). Family and dyadic patterns of functioning in maltreating families. Dans K. Browne, C. Davies, \& P. Stratton (eds), Early prediction of child abuse (pp. 161-189). New-York: Wiley.

Croake, J. W., \& Glover, K.E. (1977). A history and evaluation of parent education. Family Coordinator, 26, 151-158.

Daro, D. (1988). Confronting child abuse; Research for effective program design. NewYork: The Free Press.

Downey, G.. \& Coyne, J. (1990). Children of depressed parents: An integrative review. Psychological Bulletin, 108, 50-76. 
Fgeland, B., Breitenbucher, M., \& Rosenberg, D. (1980). Prospective study of the significance of life stress in the etiology of child abuse. Joumal of Consulting and Clinical Psychology, 48, 195-205.

Êthier, L.S. (1992). Le stress đes mères maltraitantes et leurs antécédents familiaux. Dans G. Pronovost (éd.), Comprendre la famille (pp. 645-670). Québec: Presses de I'Université đu Québec.

Ethier, L.S., Palacio-Quintin, E., \& Couture, G. (1993). Evaluation psychosociale des mères négligentes. Rapport présenté au Conseil de la santé et des services sociaux du centre du Québec (CRSSS 04).

Garbarino, J., \& Crouter, A. (1978). Defining the community context for parent-child relations: The correlates of child maltreatment. Child Development, 49, 604-616.

Gaudin, J.M. (1993). Effective intervention with neglectful families. Criminal Justice and Behavior, 20(1), 66-89.

Giovannoni, J.M. (1971). Parental mistreatment: Perpetrators and victims, Joumal of Marriage and the Family, 33, 649-657.

Hansen, D.J., Palotta, G.M. Thishelman, A.C., Conaway, L.P., \& McMillan, V.M. (1989). Parental problem-solving skills and child behavior problems. A comparison of physically abusive, neglectful, clinic and community families. Joumal of Family Violence, 4(4), 353-368.

Kimball, H., Steward, R.B., Conger, R.D., \& Burgess, R.L. (1980). A comparison of family interaction in single versus two-parent abusive, neglectful and control families. Dans T. Fields, S, Goldberg, \& A. Sostek (éds), High risk infants and children: Adult and peer interaction (pp. 43-59). New-York: Academic Press.

Kinard, E.M. (1982). Child abuse and depression: Cause or consequence? Child Welfare, 7, 403-413.

Lacharité, C. (1995, mai). Analyse des caractéristiques des figures patemelles dans les familles où les enfants sont négligés. Communication présentéc au Ve congrès de l'Association Internationale de Formation et de Recherche sur l'Éducation Familiale. Porto, Portugal.

Landsman. M.J.. Nelson, K., Allen, M., \& Tayler, M. (1992). The self-sudficiency project: Final report. Iowa City, IA: National Resource Center in Family Based Services.

Levant, R.F. (1988). Education for fatherhood. Dans P. Bronstein \& C. Pape-Cowan (éds), Fatherhood today: Men's changing role in the family (pp, 83-98). New-York: Wiley.

Lovell. M. (1986). An evaluation of a parent training and social support group for mothers at risk to maltreat their children. Thèse de doctorat inédite, University of Washington.

Palacio-Quintin, E. (1995). Les mauyais traitements envers les enfants: les facteurs sociaux et la dynamique familiale. Actes du séminaire Liens entre la violence physique, psychologique et sexuelle faite aux enfants et aux femmes (pp. 5-14). Montréal: Centre de recherche interdisciplinaire sur la violence familiale et la violence faite aux femmes, Collection Réflexions.

Palacio-Quintin, E., Couture, G., \& Paquet, J., (1995), Projet d'intervention auprès des. familles négligentes présentant ou non des comportements violents. Rapport final soumis à la Division de la prévention de la violence familiale, Santé Canada, Ottawa.

Palacio-Quintin, E., \& Desaulniers, R. (1992). Info-groupe de parent en tant que parents. Document inédit. Groupe de recherche en développement de l'enfant et de la famille, Université đu Québec à Trois-Rivières.

Palacio-Quintin, E., \& Éthier, L.S. (1993). La négligence, un phénomène négligé. Apprentissage et Socialisation, $16(1$ et 2), 153-164.

Palacio-Quintin, E., Ethier, L.S., Jourdan-Ionescu, C., \& Lacharité, C. (1995). L'intervention auprès de familles négligentes. Dans J.P. Pourtois \& H. Desmet (éd.), Blessure d'enfant (pp, 173-212). Bruxelles: De Boeck.

Polansky, N.A., Ammons, P.W., \& Gaudin, J.M. (1985). Loneliness and isolation among neglectful mothers. Social Case Work, 66, 23-36.

Polansky, N.A., Chalmers, M.A., Williams, D.P., \& Buttenwieser, E.W. (1981). Damaged parents. An anatomy of child neglect. Chicago: The University of Chicago Press. 
Price-Bonham, A.D., \& Skeen, G.H. (1979), A comparison of black and white fathers with implications for parent education. Family Coordinator, 28, 53-59.

Tavormina, J.B. (1974). Basic models of parent counseling: A critical review. Psychological Bulletin, 81, 827-835.

Twentyman, C.T., \& Bousha, D.M. (1984). Abusive, neglectful and comparison motherchild interactional style: Naturalistic observations in the home setting. Joumal of Abnormal Psychology, 93(1), 106-114.

Wolfe, D.A., Edwards, B., Manion, I., \& Koverola, C. (1988). Early intervention for parents at risk of child abuse and neglect: A preliminary investigation. Journal of Con. sulting and Clinical Psychology, 56(1), 40-47.

Wolfe, D.A., Sandler, J., \& Kaufman, K. (1981). A competency-based parent training program for child abusers. Joumal of Consulting and Clinical Psychology, 49, 633-640,

Wolock, 1., \& Horowitz, B. (1979). Child maltreatment and matemal deprivation among AFDC recipient families. Social Services Research, 53, 175-194. 